\title{
PROBABILISTIC RECURSIVE FUNCTIONS
}

\author{
BY \\ IRWIN MANN (1)
}

\begin{abstract}
The underlying question considered in this paper is whether or not the purposeful introduction of random elements, effectively governed by a probability distribution, into a calculation may lead to constructions of number-theoretic function $s$ that are not available by deterministic means. A methodology for treating this question is developed, using an effective mapping of the space of infinite sequences over a finite alphabet into itself. The distribution characterizing the random elements, under the mapping, induces a new distribution. The property of a distribution being recursive is defined. The fundamental theorem states that recursive distributions induce only recursive distributions. A function calculated by any probabilistic means is called $\psi$-calculable. For a class of such calculations, these functions are recursive. Relative to Church's thesis, this leads to an extension of that thesis: Every $\psi$-effectively calculable function is recursive. In further development, a partial order on distributions is defined through the concept of "inducing." It is seen that a recursive atom-free distribution induces any recursive distribution. Also, there exist distributions that induce, but are not induced by, any recursive distribution. Some open question s are mentioned.
\end{abstract}

I. Introduction. The theorems of Gödel and Church place crucial bounds on the possibilities of mathematical deduction and constructability. We can ask whether the notion of constructability of functions, sets, or numbers might be augmented by introducing a random process at any stage, and then consider the construction in a new, probabilistic, sense. We will show, in a sense described in $\S$ VII, that nothing new can be gained in this way that is not already available deterministically.

It is possible that such a question first occurred because of the use, in certain cases, of "Monte Carlo" methods. These concern the technique of using random sampling to obtain estimates of the answers to-even nonprobabilisticcomputational problems. The question can be construed as abstract and is mathematically relevant. One pioneering paper [de Leeuw, Moore, Shannon, and

Received by the editors August 7, 1969 and, in revised form, April 18, 1972.

AMS (MOS) subject classifications (1970). Primary 02E15, 02E99, 02F 27, 02F 35, 60B05; Secondary 02E10, 60G30, 65C05.

(1) This paper is substantially the author's doctoral dissertation, Princeton University, 1967. He wishes to express gratitude to Professor J. R. Guard and especially to Dr. Norman Shapiro for their guidance. 
Shapiro] treated and settled a particular case of part of the general theory we develop here.

We will formulate the questions and answers in the language of recursive function theory, although our notions about computing machines will often provide the motivation. The machines can be considered to be Turing machines, augmented by a random device (such as a coin, a die, or a geiger counter) that will provide an input to the machine as often as it is required. The random elements thus provided can be considered to affect the output (printed symbols on the tape) of the machine.

For the purpose of the theorems, we imagine such a machine and stochastic element generating device that gives rise to a finite or infinite sequence of output symbols. In such a general setting, it seems appropriate to consider the theory of a structure that maps arbitrarily long random sequences into other such sequences. We shall investigate the nature of these output sequences, as governed by the nature of the machine and the stochastic element generating device.

II. Preliminaries. For our purpose, we can consider sequences of elements each of which is only 0 or 1 . Let $H$ denote the space of infinite sequences of 0 's and 1's. Let $\Theta$ denote the space of finite sequences of 0 's and 1's. The empty sequence is denoted by $\varnothing$. For $\alpha \in \Theta$ and $\beta \in H \cup \Theta, \alpha * \beta$ will be the concatenation of $a$ followed by $\beta$. We have that $(*)$, for $\alpha, \beta \in \Theta$, is a recursive operation under a mapping of $\Theta \times \Theta$ to $\Theta$.

We define a partial order on $\Theta$. For $\alpha, \beta \in \Theta$, let $\alpha \leq \beta$ if there is a $\tau \in \Theta$ such that $\beta=\alpha * \pi$. This ordering can be extended to $H \cup \Theta$ by the definition that $x<y$ if and only if $x \in \Theta$ and there is a $z \in H \cup \Theta$ such that $y=x * z . x \leq y$ if and only if $x<y$ or $x=y$.

For $\theta \in \Theta$, let $|\theta|=$ length $(\theta), a(\theta)$ be the number of 1 's in $\theta$, and $b(\theta)$ be the number of 0 's in $\theta$. We have that $|\theta|, a(\theta)$, and $b(\theta)$ are recursive functions. For $x \in H \cup \Theta$, let $x_{k}$ denote the $k$ th element of $x, x^{(k)}$ denote the initial segment of $x$ of length $k$, and $x^{(0)}=\varnothing$.

Let $s(x)=\sum_{i=1}^{\infty} x_{i} \cdot 2^{-i}$. $s$ is the natural map of sequences onto real numbers in the closed interval $[0,1]$.

Let $N$ denote the set of nonnegative integers and $Q$ the rationals. There are canonical maps, recursive, $1-1$, and onto, between $\Theta, N$, and $Q$, and their finite cartesian products. Recursive functions are maps from $N^{k}$ to $N$, but we will freely speak of recursive functions from any of $\Theta, N, Q$, and their finite cartesian products to any other of the se and we will have in mind the reduction to the common case under composition with the canonical maps envisaged above.

Let $f: \Theta \rightarrow \Theta$ be a function that is monotone with respect to the ordering on $\Theta$. For $x \in H$, let $\hat{f}(x)=\sup \{f(\theta) \mid \theta<x\}$. Then $\hat{f}$ is a function from $H$ to 
$H \cup \Theta$, and it is a natural extension of $f$.

For $\theta \in \Theta$, let $H_{\theta}=\{x \in H \mid \theta<x\}$. In other words, $H_{\theta}$ is the set of elements of $H$ with initial segment $\theta$. We note that $H_{\emptyset}=H$.

III. Recursive real numbers. We shall be concerned with the class of recursive real numbers. A real number $r$ is said to be recursive if there is a recursive function $f: N \rightarrow Q$ such that $|r-f(n)|<1 / n$. This means that there are arbitrarily close, recursive, rational-valued approximations to $r$.

We have implicitly used the definition of real numbers by means of Cauchy sequences. Real numbers may also be defined by binary expansions and by Dedekind cuts, though the different theories yield the same real numbers. To each theory, there corresponds a definition of recursivity [Specker] and it is true that the different definitions yield the same class of recursive real numbers [Robinson], [Myhill]. There are, however, analytic differences. For instance, in the theory of binary real numbers, even addition is not a recursive function, while for Cauchy-recursive real numbers, every elementary function is recursive. In particular, if a recursive distribution ( $\$ I V$ below) is defined in terms of binaryrecursive real numbers, the theorems to follow will not, in general, be true.

We will, however, say for later use (Theorem $4, \S \mathrm{VI}$ ) that a binary sequence is recursive if there is a recursive function $f: N \rightarrow\{0,1\}$ such that $f(i)$ is the $i$ th place of the sequence. More generally, for arbitrary such functions $f$, the sequence is said to be recursive in $f$. And, if $x$ and $y$ are sequences, and $f$ and $g$ such functions, $y$ is said to be recursive in $x$ if $x$ is recursive in $f, y$ is recursive in $g$, and $g$ is recursive in $f$. This terminology extends to binary real numbers in the interval $[0,1]$.

The recursive real numbers form a field. The approximating rational-valued functions for sum, difference, product, and quotient are defined recursively [Rice]. We state without further proof the following lemma.

Lemma 1. If $c(\theta)$ and $d(\theta)$ are recursive real numbers, recursively enumerated by any parameter $\theta$, there exists a partial recursive function $b: \Theta \times N \rightarrow Q$ sucb that $|c(\theta) / d(\theta)-b(\theta, n)|<1 / n$ whenever $d(\theta) \neq 0$.

IV. Distributions and recursive distributions. We now characterize the random device. Consider $H$ and the $\sigma$-ring $S$ generated by the subsets $H_{\theta}$ of $H$. We will consider any probability measure $\psi$ on $S$ as a measure on $H$, and throughout this paper, we will treat only such measures and refer to them simply as distributions on $H$. A measurable set, then, will be simply an element of $S$. We note that $H$ is a member of $S$ because $H=H_{\emptyset}$ and $\psi\{H\}=1$.

Definition. Let $\psi$ be a measure on $H$. We will say $\psi$ is a recursive distribution on $H$ if there is a recursive function $f: \Theta \times N \rightarrow Q$ such that $\mid \psi\left\{H_{\theta}\right\}-$ $f(\theta, n) \mid<1 / n$. 
It is an immediate consequence of the definition that if $\psi$ is recursive, then $\psi\left\{H_{\theta}\right\}$ is a recursive real number for all $\theta \in \Theta$.

Throughout the discussion, we have in mind the possibility of computation. When a distribution is recursive, it means that it is possible by using a standard method to choose random elements according to the distribution. In fact, this can be done by repeated tosses (along with some calculation) of a fair coin, or a suitable simulation of them. Theorem 7 ( $\$$ VIII) makes this statement precise. Further, it may not be surprising that recursive distributions have considerable structure. To use the natural phraseology, the "bits" of a generated random element $\theta$ may be "independent" with identical or differing probabilities (such as a coin that comes up heads on the $i$ th toss with probability $1 / i$ ), or they may be correlated (which in turn gives rise to the possibility of having certain infinite sequences occur with positive probability).

There is a particular distribution of interest. For $\theta \in \Theta$ and $p$ a real number with $0<p<1$, and recalling that $a(\theta)$ and $b(\theta)$ are the number of 1 's and 0 's, respectively, in $\theta$, let $\psi_{p}\left\{H_{\theta}\right\}=p^{a(\theta)}(1-p)^{b(\theta)}$. It is well known that $\psi_{p}$ is a measure (see for instance [Halmos, p. 157, Theorem B], taking $H$ to be the cartesian product of countably many two-point spaces).

Theorem 1. Let $p$ be a real number with $0<p<1 . \psi_{p}$ is recursive if and only if $p$ is recursive.

Proof. Suppose $p$ is recursive. Then there is a recursive function $f: N \rightarrow Q$ such that $|p-f(n)|<1 / n . \psi_{p}\left\{H_{\theta}\right\}=p^{a(\theta)}(1-p)^{b(\theta)}$. This is a polynomial in $p$, and on the closed interval $[0,1]$, its derivative has the upper bound $a(\theta)+$ $b(\theta)=|\theta|$. Then, by the law of the mean,

$$
\left|\psi_{p}\left\{H_{\theta}\right\}-\psi_{p *}\left\{H_{\theta}\right\}\right| \leq|\theta| \cdot\left|p-p^{*}\right|
$$

for all $p^{*} \in[0,1]$. Now let $p^{*}(n)=\max [0, \min [f(n \cdot|\theta|), 1]]$. Then

$$
g(\theta, n) \stackrel{\text { def }}{=} \psi_{p *(n)}\left\{H_{\theta}\right\}=p^{*}(n)^{a(\theta)}\left[1-p^{*}(n)\right]^{b(\theta)}
$$

is a recursive function as $a(\theta), b(\theta)$, and $p^{*}(n)$ are. As $\left|p-p^{*}(n)\right|<1 /(n \cdot|\theta|)$, we have $\left|\psi_{p}\left\{H_{\theta}\right\}-g(\theta, n)\right|<|\theta| \cdot 1 /(n \cdot|\theta|)=1 / n$, and hence $\psi_{p}$ is recursive. We have proved one part of the the orem.

Conversely, let $\psi_{p}$ be recursive. For the finite sequence $\theta=1, \psi_{p}\left\{H_{\theta}\right\}=$ $p$. Then $p$ is recursive. This completes the proof.

V. Admissibility and induced distributions. We now make precise the nature of the stochastic recursive calculation.

Definition. A pair $(\psi, f)$ where $\psi$ is a distribution on $H$ and $f: \Theta \rightarrow \Theta$ is a function, is called admissible if: 
(1) $f$ is recursive and monotone,

(2) for $\theta \in \Theta, H_{\theta}$ is measurable and $\psi\{H\}=1$, and

(3) $\psi\{x \in H \mid \hat{f}(x) \in H\}=1$.

The third condition for admissibility insures that almost all infinte sequences are mapped by $\hat{f}$ into infinite sequences. It is analogous for stochastic calculation to the notion that a function is total, rather than partial, recursive. Subsequently (in $\$ \mathrm{VI}$ ), we will give an example to show that the fundamental theorem does not hold if the restriction is not there.

An admissible pair characterizes the mathematical "software" of a machine random device combination. If $\psi$ is recursive, the admissible pairs $(\psi, f)$ represent stochastic analogues of effective computability. But we note that even when $\psi$ and $f$ are both recursive, the pair $(\psi, f)$ may not be admissible.

For a definition to follow, we will need a lemma.

Lemma 2. If $H^{*} \subseteq H$ and $H^{*}$ is measurable, and $f: \Theta \rightarrow \Theta$ is monotone, then $\hat{f}^{-1}\left(H^{*}\right)$ is measurable.

Proof. We show first that for $\theta \in \Theta, \hat{f}^{-1}\left(H_{\theta}\right)$ is measurable. We have $\hat{f}^{-1}\left(H_{\theta}\right)=\left\{x \mid \lim _{n \rightarrow \infty} f\left(x^{(n)}\right) \in H_{\theta}\right\}=\left\{x \mid(\exists i)\left[f\left(x^{(i)}\right) \geq \theta\right]\right\}=\bigcup_{i=1}^{\infty}\left\{x \mid f\left(x^{(i)}\right) \geq \theta\right\}$.

Now for any $i,\left\{x \mid f\left(x^{(i)}\right) \geq \theta\right\}$ is measurable, because it is the union of at most $2^{i}$ sets $H_{a}$ with $|a|=i$. Then $\hat{f}^{-1}\left(H_{\theta}\right)$ is a countable union of measurable sets, hence measurable.

Now let $C=\left\{X \mid \hat{f}^{-1}(X)\right.$ is measurable $\}$. It is easily seen that $C$ is a $\sigma$-field. $C$ contains $H_{\theta}$ for all $\theta \in \Theta$, and hence contains any element in the $\sigma$-field $S$ generated by the $H_{\theta}$. Since $H^{*}$ is measurable, $H^{*} \in S$. Hence $H^{*} \in C$, and $\hat{f}^{-1}\left(H^{*}\right)$ is measurable. This proves the lemma.

Given any admissible pair $(\psi, f)$, we will speak of the distribution induced by $(\psi, f)$.

Definition. The distribution $\phi$ given by

$$
\phi\left\{H^{*}\right\}=\psi\left\{\hat{f}^{-1}\left(H^{*}\right)\right\}
$$

for $H^{*} \subseteq H$ and $H^{*}$ measurable is said to be induced by an admissible pair $(\psi, f)$, and we say that $(\psi, f)$ induces $\phi$. If there is a function $f$ such that $(\psi, f)$ is admissible and induces $\phi$, we say the $\psi$ induces $\phi$.

Because of Lemma 2, the induced distribution is well-defined. A machinerandom device thus induces a new distribution.

Theorem 2. If $\psi_{1}, \psi_{2}$, and $\psi_{3}$ are distributions on $H$ such that $\psi_{1}$ induces $\psi_{2}$ and $\psi_{2}$ induces $\psi_{3}$, then $\psi_{1}$ induces $\psi_{3}$. 
Proof. Let $\left(\psi_{1}, f_{12}\right)$ and $\left(\psi_{2}, f_{23}\right)$ be admissible pairs that induce $\psi_{2}$ and $\psi_{3}$ respectively. We shall show that there exists a function $f_{13}: \Theta \rightarrow \Theta$ such that: (a) $f_{13}$ is recursive, (b) $f_{13}$ is monotone, (c) $\left(\psi_{1}, f_{13}\right)$ is admissible, and (d) $\left(\psi_{1}, f_{13}\right)$ induces $\psi_{3}$.

Let $f_{13}=f_{23}\left(f_{12}\right)$.

(a) $f_{13}$ is recursive as it is a composition of recursive functions.

(b) Let $\alpha \leq \beta$. Then $f_{12}(\alpha) \leq f_{12}(\beta)$. $f_{13}(\alpha)=f_{23}\left[f_{12}(\alpha)\right] \leq f_{23}\left[f_{12}(\beta)\right]=$ $f_{13}(\beta)$ and $f_{13}$ is monotone.

(c) We must show that $\psi\left\{x \in H \mid \hat{f}_{13}(x) \in H\right\}=1$. We have

$$
\begin{array}{r}
\psi_{1}\left\{x \in H \mid \hat{f}_{13}(x) \in H\right\}=\psi_{1}\left\{\hat{f}_{13}^{-1}(H)\right\}=\psi_{1}\left\{\left[\hat{f}_{23}\left(\hat{f}_{12}\right)\right]^{-1}(H)\right\} \\
=\psi_{1}\left\{\left[\hat{f}_{12}^{-1}\left(\hat{f}_{23}^{-1}\right)\right](H)\right\}=\psi_{2}\left\{\hat{f}_{23}^{-1}(H)\right\}=\psi_{3}\{H\}=1 .
\end{array}
$$

(d) We show that for each measurable $H^{*} \subseteq H, \psi_{3}\left\{H^{*}\right\}=\psi_{1}\left\{\hat{f}_{13}^{-1}\left(H^{*}\right)\right\}$. We have

$\psi_{3}\left\{H^{*}\right\}=\psi_{2}\left\{\hat{f}_{23}^{-1}\left(H^{*}\right)\right\}=\psi_{1}\left\{\hat{f}_{12}^{-1}\left[\hat{f}_{23}^{-1}\left(H^{*}\right)\right]\right\}=\psi_{1}\left\{\left[\hat{f}_{23}\left(\hat{f}_{12}\right)\right]^{-1}\left(H^{*}\right)\right\}=\psi_{1}\left\{\hat{f}_{13}^{-1}\left(H^{*}\right)\right\}$.

This proves the theorem.

VI. The fundamental theorem. We come now to the fundamental theorem.

Theorem 3. Any distribution induced by a recursive distribution is recursive.

Proof. $(\psi, f)$ is an admissible pair and induces. $\phi$. We must consider $\phi\left\{H_{\theta}\right\}$. With respect to a given $\theta \in \Theta$, we define sets:

$$
A(\theta)=\{\sigma \in \Theta \mid \theta \leq f(\sigma)\}, \quad B(\theta)=\{\sigma \in \Theta \mid f(\sigma)<\theta\} .
$$

We note that there may be $\sigma \in \Theta$ such that $f(\sigma)$ is not comparable with $\theta$. Define

We have

$$
y_{k}(\theta)=\sum_{|\sigma|=k ; \sigma \epsilon A(\theta)} \psi\left\{H_{\sigma}\right\} \text { and } z_{k}(\theta)=\sum_{|\sigma|=\sum_{k ; \sigma \epsilon B(\theta)}} \psi\left\{H_{\sigma}\right\} .
$$

$$
\begin{aligned}
y_{k}(\theta) & =\psi\left\{x \in H \mid \theta \leq f\left(x^{(k)}\right)\right\} \leq \psi\left\{\bigcup_{i=1}^{\infty}\left\{x \in H \mid \theta \leq f\left(x^{(i)}\right)\right\}\right\} \\
& =\psi\left\{x \in H|(\exists i)| \theta \leq f\left(x^{(i)}\right) \mid\right\}=\psi\left\{\hat{f}^{-1}\left(H_{\theta}\right)\right\}=\phi\left\{H_{\theta}\right\} \\
& =\psi\left\{x \in H \mid(\exists i)\left[\theta \leq f\left(x^{(i)}\right)\right]\right\} \leq \psi\left\{x \in H \mid \theta \leq f\left(x^{(k)}\right) \text { or } f\left(x^{(k)}\right)<\theta\right\} \\
& =\psi\left\{x \mid \theta \leq f\left(x^{(k)}\right)\right\}+\psi\left\{x \mid f\left(x^{(k)}\right)<\theta\right\}=y_{k}(\theta)+z_{k}(\theta) .
\end{aligned}
$$

We have $y_{k}(\theta) \leq \phi\left\{H_{\theta}\right\} \leq y_{k}(\theta)+z_{k}(\theta)$. We show first that $\lim _{k \rightarrow \infty} z_{k}(\theta)=0$. Define the set

$$
N_{k}(\theta)=\left\{x \in H \mid f\left(x^{(k)}\right)<\theta\right\}
$$

$N_{k}(\theta)$ is measurable as it is the union of at most $2^{k}$ measurable sets. 
$N_{k+1}(\theta) \subseteq N_{k}(\theta)$. Let $N(\theta)=\bigcap_{k=1}^{\infty} N_{k}(\theta) . N(\theta)$ is measurable. Since $\psi$ is a finite measure, we have [Halmos, p. 38, Theorem E]

$$
\lim _{k \rightarrow \infty} \psi\left\{N_{k}(\theta)\right\}=\psi\{N(\theta)\} \text {. }
$$

But we have

$$
N(\theta)=\{x \in H \mid \hat{f}(x)<\theta\} \subseteq\{x \in H \mid \hat{f}(x) \text { is finite }\} .
$$

$N(\theta)$ is measurable. Then because $(\psi, f)$ is admissible and because a measurable subset of a set of measure 0 has measure $0, \psi\{N(\theta)\}=0$. But $z_{k}(\theta)=$ $\psi\left\{N_{k}(\theta)\right\}$. Then $\lim _{k \rightarrow \infty} z_{k}(\theta)=0$.

We have that $\phi\left\{H_{\theta}\right\}=\lim _{k \rightarrow \infty} y_{k}(\theta)$. We must show that $\phi$ is recursive.

Since $\psi$ is recursive, there is a recursive function $g: \Theta \times N \rightarrow Q$ such that, for $\sigma \in \Theta,\left|\psi\left\{H_{\sigma}\right\}-g(\sigma, n)\right|<1 / n$. Let $\hat{\psi}_{k}\left\{H_{\sigma}\right\}=g\left(\sigma, k \cdot 2^{k}\right)$. Then $\mid \psi\left\{H_{\sigma}\right\}-$ $\hat{\psi}_{k}\left\{H_{\sigma}\right\} \mid<1 /\left(k \cdot 2^{k}\right)$.

Define

$$
\hat{y}_{k}(\theta)=\sum_{|\sigma|=k ; \sigma \in A(\theta)} \hat{\psi}_{k}\left\{H_{\sigma}\right\}
$$

and

$$
\hat{z}_{k}(\theta)=\sum_{|\sigma|=\sum_{k} ; \sigma \in B(\theta)} \hat{\psi}_{k}\left\{H_{\sigma}\right\}
$$

We have

$$
\begin{aligned}
\left|y_{k}(\theta)-\hat{y}_{k}(\theta)\right| & =\left|\sum_{|\sigma|=k ; \sigma \in A(\theta)}\left[\psi\left\{H_{\sigma}\right\}-\hat{\psi}_{k}\left\{H_{\sigma}\right\}\right]\right| \\
& \leq \sum_{|\sigma|=k ; \sigma \in A(\theta)}\left|\psi\left\{H_{\sigma}\right\}-\hat{\psi}_{k}\left\{H_{\sigma}\right\}\right|<2^{k} \cdot 1 /\left(k \cdot 2^{k}\right)=1 / k .
\end{aligned}
$$

Similarly, $\left|z_{k}(\theta)-\hat{z}_{k}(\theta)\right|<1 / k$.

Since $y_{k}(\theta) \leq \phi\left\{H_{\theta}\right\} \leq y_{k}(\theta)+z_{k}(\theta)$, we have $\hat{y}_{k}(\theta)-1 / k<\phi\left\{H_{\theta}\right\}<\hat{y}_{k}(\theta)+$ $\hat{z}_{k}(\theta)+2 / k$. Then $\left|\phi\left\{H_{\theta}\right\}-\hat{y}_{k}(\theta)\right|<\left|\hat{z}_{k}(\theta)\right|+2 / k$. Let

$$
k^{*}(\theta, n)=(\mu k)\left[\left|\hat{z}_{k}(\theta)\right|+2 / k \leq 1 / n\right]
$$

where $\mu$ denotes minimalization. $\hat{y}_{k}(\theta)$ and $\hat{z}_{k}(\theta)$ are recursive in $k$ and $\theta$. $\lim _{k \rightarrow \infty} \hat{z}_{k}(\theta)=0$. Hence $k^{*}(\theta, n)$ is recursive. Define $b(\theta, n)=\hat{y}_{k^{*}(\theta, n)}(\theta)$; $b(\theta, n)$ is recursive and $\left|\phi\left\{H_{\theta}\right\}-b(\theta, n)\right|<1 / n$. This proves the theorem.

We pause in the principal line of development in the paper to reconsider the restriction in the definition of admissibility, namely:

$$
\psi\{x \in H \mid \hat{f}(x) \in H\}=1 \text {. }
$$

It might be that, without the restriction, The orem 3 suitably generalized still holds. This possibility is removed by the following counterexample. 
We will choose a recursive and monotone function $f$ such that

$$
\psi_{1 / 2}\{x \in H \mid \hat{f}(x) \in H\}<1 .
$$

$\psi_{1 / 2}$ is recursive, $\left(\psi_{1 / 2}, f\right)$ will be otherwise admissible, and the induced distribution $\phi$ will not be recursive. In particular, $\phi\{H\}$ will not be a recursive real number.

Let $g: N \rightarrow N$ be a recursive function such that $g(N)$ is a nonrecursive set. Let $b$ be the characteristic function of $g(N)$. Let $y=\sum_{i=1}^{\infty} 2^{-b(i)} . y$ is a nonrecursive real number.

For $x \in H \cup \Theta$, let $s(x)=\sum_{i=1}^{|x|} 2^{-x_{i}}$. We will find a recursive, monotone function $f: \Theta \rightarrow \Theta$ such that

$$
f(x)= \begin{cases}x & \text { if } s(x) \leq y, \\ \varnothing & \text { if } s(x)>y,\end{cases}
$$

where $\varnothing$ has length 0 . Then

$$
\begin{aligned}
\phi\{H\} & =\psi_{1 / 2}\{x \in H \mid \hat{f}(x) \in H\}=\psi_{1 / 2}\{x \in H \mid \hat{f}(x)=x\} \\
& =\psi_{1 / 2}\{x \in H \mid s(x) \leq y\}=y .
\end{aligned}
$$

In the last equality, we use the fact that $\psi_{1 / 2}$ and $s$ together yield Borel measure. Also, we note that a number nonrecursive in the binary sense is nonrecursive in our sense.

Then $\phi\{H\}=\phi\left\{H_{\emptyset}\right\}$ is nonrecursive and hence $\phi$ is nonrecursive.

Finally, we fashion the function $f$. Let

where

$$
\Omega=\left\{\theta \in \Theta \mid s(\theta)<\sum_{k \in S(\theta)} 2^{-k}\right\}
$$

$$
S(\theta)=\left\{k \in N \mid(\exists j)_{\leq|\theta|}[g(j) \leq|\theta| \text { and } k=g(j)]\right\} .
$$

For $\theta \in \Omega, s(\theta)<y . \Omega$ is a recursive set. If $\alpha \in \Omega$ and $\alpha \leq \beta$, then $\beta \in \Omega$. In other words, $\Omega$ is hereditary. Let

$$
f(\theta)= \begin{cases}\theta & \text { if } \theta \in \Omega, \\ \varnothing & \text { if } \theta \notin \Omega .\end{cases}
$$

$f$ is recursive because $\Omega$ is recursive and $f$ is monotone because $\Omega$ is hereditary. Our example is complete.

We proceed now in the general development.

Definition. $x \in H$ is an atom of a distribution $\psi$ if $\psi\{x\}>0$.

The significance of the following theorem is that, while it would follow that $\psi\{x\}$ is recursive for recursive $\psi$, we will show that $x$ itself is recursive. 
Theorem 4. Every atom of a recursive distribution is recursive.

Proof. Let $x$ be an atom of a recursive distribution $\psi$. Let

$$
r(i)=\psi\left\{H_{x}^{(i+1)}\right\} / \psi\left\{H_{x}^{(i)}\right\} .
$$

$\psi\left\{H_{x(i)}\right\} \neq 0$, since $x$ is an atom. $r(i)$ is the conditional probability of the next bit of the atom "occurring" after $i$ bits have already occurred.

Since $\psi\{x\}=\prod_{i=1}^{\infty} r(i)$, we have $\lim _{i \rightarrow \infty} r(i)=1$, for otherwise $x$ could not be an atom. Then there is an $i_{0}$ such that for $i>i_{0}, r(i)>3 / 4$. From this it follows that for $i>i_{0}$, one of the following pair of inequalities holds:

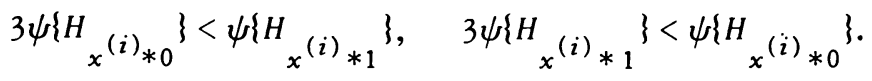

Now, since $\psi$ is recursive, there is a recursive function $g: \Theta \times N \rightarrow Q$ such that $\left|\psi\left\{H_{\theta}\right\}-g(\theta, n)\right|<1 / n$. For sufficiently large $n$, we have that either

$$
3 g\left(x^{(i)} * 0, n\right)<g\left(x^{(i)} * 1, n\right)+4 / n
$$

or

$$
3 g\left(x^{(i)} * 1, n\right)<g\left(x^{(i)} * 0, n\right)+4 / n
$$

but not both. Let $n^{*}(i)$ be the minimum $n$ for which that is true. $n^{*}(i)$ is a recursive function of $i$.

Then $x=x^{\left(i_{0}\right)} * y$ where

$$
y_{k}= \begin{cases}0 & \text { if } R(k) \text { holds } \\ 1 & \text { otherwise }\end{cases}
$$

and $R(k)$ is

$$
\begin{aligned}
g\left[x^{\left(i_{0}\right)} * y_{k-1} * 0, \quad n^{*}\left(i_{0}+k-1\right)\right]+4 / n *\left(i_{0}+k-1\right) & \\
& >3 g\left[x^{\left(i_{0}\right)} * y_{k-1} * 1, n *\left(i_{0}+k-1\right)\right] .
\end{aligned}
$$

Then $x$ is a finite sequence concatenated $w$ ith a sequence that is defined in terms of cases each of which is recursive. Therefore $x$ is recursive. This completes the proof.

We include the following corollary already in the literature.

Corollary [de Leeuw, Moore, Shannon, and Shapiro]. Suppose $p$, with $0<$ $p<1$, is recursive, $\psi_{p}$ induces $\phi$, and $x$ is an atom of $\phi$. Then $x$ is recursive.

Proof. Since $p$ is recursive, $\psi_{p}$ is recursive. Since $\psi_{p}$ induces $\phi, \phi$ is recursive. Then $x$ is recursive.

This a principal result in the above mentioned paper [Theorem 3, p. 193]. It is included here to demonstrate the power of the general methods. 
Actually, a somewhat stronger result is obtained in the previous paper: Suppose $p$ is any real number with $0<p<1, \psi_{p}$ induces $\phi$, and $x$ is an atom of $\phi$. Then $x$ is recursive in $p$.

VII. Calculability of functions. We return to the question raised earlier that asked whether a construction (most generally, that of evaluating a function), not available deterministically, might exist through the possibility of probabilistic calculation.

Through the correspondence of Turing machines with recursive functions, we envisage a machine corresponding to a function $g$ and the machine augmented with a random device characterized by a distribution $\psi$. Let $(\psi, g)$ induce $\phi$. We will describe what it might mean to calculate a function $f$ with such a machine.

The output is an infinite sequence $x \in H$. There is a natural identification of sequences and functions. Let $x^{\sharp}(n)$ denote the number of successive 1's immediately following the $n$ th. 0 of $x$. If there is not an $n$th 0 , the function $x^{\#}(n)$ is undefined for $n$. The particular identification employed here is not the only one that is possible. But any other scheme, for total functions, can be reduced to this by composition with $g$.

Let $H^{\#}=\{x \in H \mid x$ contains an infinite number of 0 's $\} . H^{\#}$ denotes the set of sequences for which $x^{\sharp}(n)$ is defined for all $n$. It is measurable and we will say that $\phi$ is proper if $\phi\left\{H^{\#}\right\}=1$. This is analogous to the similar condition for admissibility. It is a necessary condition for computation. In this section we will suppose that $\phi$ is proper.

Although $x^{\sharp}(n)$ is determined by $x, x$ itself is stochastic. We must prescribe the définition of $f$. Let

$$
w_{\phi}(n, m)=\phi\left\{x \in H \mid x^{\#}(n)=m\right\} .
$$

We consider three functions that can be defined:

(1) $f^{*}(n)=m$. if $w_{\phi}(n, m)>1 / 2$,

(2) $f^{* *}(n)=m$ if $w_{\phi}(n, m)>w_{\phi}(n, i)$ for all $i \neq m$,

(3) $f^{* * *}(n)=m$ if $w_{\phi}(n, m)>0$ and $m=\min _{j}\left[w_{\phi}(n, j) \geq w_{\phi}(n, i)\right.$ for all $\left.i\right]$. We have that domain $\left(f^{*}\right) \subseteq \operatorname{domain}\left(f^{* *}\right) \subseteq \operatorname{domain}\left(f^{* * *}\right)$.

These are the weakest definitions we can imagine to impart to the notion of a function being calculable by probabilistic means. For every proper distribution $\phi$, these functions are defined. Conversely, if a function is to be calculable in the probabilist ic sense, it must be possible to produce such a $\phi$. We leave the method of doing this unspecified.

We indicate the nature of these functions when $\phi$ is recursive, before we give the proofs below. The demonstration is included there that if $\phi$ is recursive, there are recursive rational approximations to $w_{\phi}(n, m)$. 
(1) For given $n$, we examine $w_{\phi}(n, m)$ for successive candidates for $m$, $m=0,1,2, \ldots$. If there is an $m$, it is found recursively as the one for which $w_{\phi}(n, m)$ is bounded above $1 / 2$. Then the function $f^{*}$ is partial recursive.

(2) For given $n$, we consider the first $k$ candidates for $m$ together. If there is an $m$, there comes a sufficiently large $k$ such that what remains out of 1 from the sum of the $w_{\phi}(n, i), i=0, \cdots, k$, so far, is less than the largest $w_{\phi}(n, i)$ that has already appeared. Then $m$ is among the first $k$ candidates, and if there is a largest, it may be found recursively. Hence $f^{* *}$ is partial recursive.

(3) For given $n$, we again examine candidates for $m$ such that $w_{\phi}(n, m)$ is maximum. If this maximum is shared by more than one candidate, we discriminate among the arguments of $w_{\phi}(n, m)$ to choose the minimum. But this case is different, as it is not a recursive process to determine if two recursive real numbers are equal. It follows that computation, in general, is not feasible. We will include an example below to show that $f^{* * *}$ need not be recursive.

We call a function $\psi$-calculable if it is obtained by the probabilistic means given by (1) or (2).

We proceed to prove the statements we have made.

Theorem 5. If $\phi$ is a recursive proper distribution on $H$ and

$$
w_{\phi}(n, m)=\phi\left\{x \in H \mid x^{\sharp}(n)=m\right\},
$$

then

(a) if $f^{*}(n)=(\mu m)\left[w_{\phi}(n, m)>1 / 2\right]$, then $f^{*}$ is a partial recursive function;

(b) if $f^{* *}(n)=(\mu m)\left[u_{\phi}(n, m)>w_{\phi}(n, i)\right.$ for all $\left.i \neq m\right]$, then $f^{* *}$ is a partial recursive function.

Proof. We show first that there is a recursive function $\hat{w}: N \times N \times N \rightarrow Q$ such that $\left|w_{\phi}(n, m)-\hat{w}(n, m, k)\right|<1 / k$. Let $A_{n, m}=\{\theta \in \Theta \mid \theta$ is the initial segment of a sequence $x \in H$ having least length such that necessarily $\left.x^{\sharp}(n)=m\right\}$. Then, for all $x \in H^{\#}, x^{\#}(n)=m$ if and only if there is an $i$ such that $x^{(i)} \in A_{n, m}$; and if $\alpha, \beta \in A_{n, m}$ and $\alpha \neq \beta$, then $H_{\alpha}$ and $H_{\beta}$ are disjoint. Then $\left\{x \in H^{\sharp} \mid x^{\sharp}(n)\right.$ $=m\}$, as the union of a countable number of $H_{\theta}$, is measurable, and $w_{\phi}(n, m)=$ $\Sigma_{\theta \in A_{n, m}} \phi\left\{H_{\theta}\right\}$.

Since $\phi$ is recursive, there is a recursive function $b: \Theta \times N \rightarrow Q$ such that $\left|\phi\left\{H_{\theta}\right\}-b(\theta, k)\right|<1 / k$. Also, $\theta \in A_{n, m}$ is a recursive relation in $\theta, n$, and $m$. The number of $\theta$ such that $|\theta| \leq k$ is $2^{k+1}-1$. Let

$$
u(n, m, k)=\sum_{\theta \in A_{n, m} ;|\theta| \leq k}\left[b\left(\theta, 2 k \cdot 2^{k+1}\right)-\frac{1}{2 k \cdot 2^{k+1}}\right] .
$$

We have that $u(n, m, k) \leq w_{\phi}(n, m)$. Also 


$$
w_{\psi}(n, m) \leq 1-\sum_{j=0 ; j \neq m}^{k} u\left(n, j, 2 k^{2}\right) \stackrel{\text { def }}{=} v(n, m, k),
$$

where $u$ and $v$ are recursive, and converge in $k$ to $w_{\phi}(n, m)$. Let

$$
\hat{k}(n, m, k)=\left(\mu k^{*}\right)\left[v\left(n, m, k^{*}\right)-u\left(n, m, k^{*}\right)<1 / k\right] .
$$

Then let $\hat{w}(n, m, k)=u[n, m, \hat{k}(n, m, k)]$. We have shown that $w_{\phi}(n, m)$ is recursively approximated by $\hat{w}(n, m, k)$.

We now complete the proof of the theorem.

(a) $f^{*}(n)=K[(\mu z)\{\hat{w}(n, K(z), L(z))>1 / 2+1 / L(z)\}]$ where $J(x, y)=z, K(z)$ $=x$, and $L(z)=y$ are recursive pairing functions. Then $f^{*}$ is partial recursive, as there is at most one $m \in N$ such that $w_{\phi}(n, m)>1 / 2$.

We note that (a) is also true where $r$ is any recursive real number with $r>$ $1 / 2$, and $r$ replaces $1 / 2$ in the above.

(b) Define the relation $R(n, m, k)$ on $N \times N \times N$ to be the conjunction of

$$
\hat{w}(n, m, k)>\hat{w}(n, j, k)+2 / k \quad \text { for all } j \neq m \text { and } j \leq k
$$

and

$$
\hat{w}(n, m, k)>1-\sum_{j=0}^{k} \hat{w}\left(n, j, k^{2}\right)+2 / k .
$$

$R(n, m, k)$ is a recursive relation.

It follows that if $R(n, m, k)$ holds for some $m \geq 0$ and $k \geq 1$, then $f^{* *}(n)$ $=m$, and if $\sim R(n, m, k)$ holds for all $m \geq 0$ and $k \geq 1$, then $f^{* *}(n)$ is undefined.

$$
f^{* *}(n)=K[(\mu z) R(n, K(z), L(z))]
$$

where $J(x, y)=z, K(z)=x$, and $L(z)=y$ are again the pairing functions. Then $f^{* *}$ is partial recursive. This proves the theorem.

We now give the example showing that $f^{* * *}$ need not be recursive.

Example. There is a recursive, proper distribution $\phi$ such that

$$
f^{* * *}(n)=\min _{j}\left[w_{\phi}(n, j)>0 \text { and } w_{\phi}(n, j) \geq w_{\phi}(n, i) \text { for all } i\right]
$$

and $f^{* * *}$ is total, binary-valued, and nonrecursive.

Proof. We denote the positive integers by $N^{+}$. Let $R(n, m)$ be a recursive relation on $N^{+} \times N^{+}$such that $(\exists m) R(n, m)$ is not recursive on $N^{+}$. We will produce a $\phi$ such that

where

$$
x^{\sharp}(n)= \begin{cases}0 & \text { with probability } v(n), \\ 1 & \text { otherwise }\end{cases}
$$

$$
v(n)=\left\{\begin{array}{l}
1 / 2-1 / 2 m^{*}(n) \text { if }(\exists m) R(n, m), \\
1 / 2 \text { if } \sim(\exists m) R(n, m)
\end{array}\right.
$$


and $m^{*}(n)=(\mu m) R(n, m)$.

Then

$$
f * * *(n)= \begin{cases}0 & \text { if } \sim(\exists m) R(n, m), \\ 1 & \text { if }(\exists m) R(n, m),\end{cases}
$$

in other words, $f^{* * *}$ is the characteristic function of $\sim(\exists m) R(n, m)$.

$v(n)$ has the rational approximations

$$
b(n, k)= \begin{cases}1 / 2-1 / 2 m^{*}(n) & \text { if }(\exists m)_{\leq k} R(n, m), \\ 1 / 2-1 / 2 k & \text { if } \sim(\exists m)_{\leq k} R(n, m),\end{cases}
$$

such that $|v(n)-b(n, k)|<1 / k . \quad b$ is recursive because $R$ is recursive.

Let $\psi$ be a distribution on $H$ such that $\psi\left\{x \in H \mid x_{n}=0\right\}=v(n)$. A measure that has this property is defined by

$$
\psi\left\{H_{\theta}\right\}=\left[\prod_{n=1 ; \theta_{n}=0}^{|\theta|} v(n)\right]\left[\prod_{n=1 ; \theta_{n}=1}^{|\theta|}(1-v(n))\right] \quad \text { for } \theta \in \Theta .
$$

As in the proof of Theorem 1, $\psi$ can be shown to be recursive by means of $b(n, k)$.

We will find $g$ such that $(\psi, g)$ induces $\phi$.

There is a natural 1-1 correspondence between $x \in H$ and functions $\bar{x}$ : $N^{+} \rightarrow\{0,1\}$ by means of $\bar{x}(i)=x_{i}$. For any $x \in H$, there is a unique $y \in H$ such that $y^{\#}(0)=0$ and $y^{\#}=\bar{x}$. Define $G: H \rightarrow H$ such that $[G(x)]^{\#}=\bar{x}$. Then there is a recursive, monotone function $g: \Theta \rightarrow \Theta$ such that $\hat{g}=G$. Let $(\psi, g)$ induce $\phi$. It follows that

(1) $\hat{g}(H) \subseteq H^{\#}$,

(2) $\hat{g}(H)=\left\{x \in H^{\#} \mid x^{\#}\right.$ is binary valued $\}$,

(3) $\phi\left\{H^{\#}\right\}=1$,

(4) $\phi\left\{x \in H^{\#} \mid x^{\#}\right.$ is binary valued $\}=1$,

(5) $\phi\left\{x \in H^{\#} \mid x^{\#}(n)=0\right\}=v(n)$.

Hence $f^{* * *}$ is total, binary valued, and nonrecursive, although $\phi$ is a recursive, proper distribution. This completes the example. It is interesting to note that in this case $f^{* *}$ agrees with $f^{* * *}$ where $f^{* *}$ is defined, and it is identically 1 .

We turn now to the notion of effective calculability. As in the deterministic case, there must exist an algorithm for the calculation-effective in the sense that there is a finite description that enables us to calculate any specific value of the function, the same description to cover all arguments in the domain of the function. Church's the sis, enunciated in 1936 [Church], suggests that the only effectively calculable functions are recursive. Now extend this (necessarily informal) meaning of algorithm to include the possible introduction of random 
elements (that is, the description of their realization). Relative to Church's thesis, such an algorithm can mean only an admissible pair $(\psi, g)$ where $\psi$ is recursive. We will call a function $\psi$-effectively calculable if there is any algorithm at all, in general probabilistic, that yields the function.

Church's thesis is not a statement about stochastic algorithms and would not have been infringed upon even if the results of this paper were contrary to the ones actually obtained. And perhaps these results are not surprising. Yet in a sense, they do provide, relative to the thesis, an extension of it: Every $\psi$ effectively calculable function is recursive.

VIII. A partial order on distributions. We begin the exploration of the character of distributions that induce and are induced by other distributions.

Theorem 6. For all $p$ with $0<p<1$, (a) $\psi_{p}$ is atom-free, (b) if $\psi$ induces $\psi_{p}$, then $\psi$ is atom-free.

Proof. (a) For $y \in H$,

$$
\psi_{p}\{y\}=\lim _{k \rightarrow \infty} p^{a\left(y^{(k)}\right)}(1-p)^{b\left(y^{(k)}\right)}=0 .
$$

Then $y$ cannot be an atom of $\psi_{p}$.

(b) Suppose, to the contrary, that $\psi$ induces $\psi_{p}$ and $x$ is an atom of $\psi$. Then there is a recursive function $f: \Theta \rightarrow \Theta$ such that $(\psi, f)$ induces $\psi_{p}$. Let $y=f(x)$. Then $y$ is an atom of $\psi_{p}$. Hence $x$ cannot be an atom of $\psi$.

This proves the theorem. We note that the proof of (b) holds as well for any atom-free measure $\phi$ in place of $\psi_{p}$.

We see that if $\psi$ and $\phi$ are both recursive, it is not necessarily true that $\psi$ induces $\phi$. It may be that $\psi$, though recursive, is not "smooth" enough-it may have atoms. We can show, however, that if $\phi$ is recursive, there is a nontrivial class of distributions that induce it.

Theorem 7. If $\phi$ is a recursive distribution on $H$, then $\psi_{1 / 2}$ induces $\phi$.

Proof. In what follows, $a \in \Theta$ is an initial segment of $x$, the input sequence. $\theta \in \Theta$ is an initial segment of the output sequence. Let

$$
r(\theta)=\left\{\begin{array}{l}
\phi\left\{H_{\theta * 0}\right\} / \phi\left\{H_{\theta}\right\} \text { if } \phi\left\{H_{\theta}\right\} \neq 0, \\
0 \text { otherwise, }
\end{array}\right.
$$

where $r(\theta)$ is the conditional probability $\phi\left\{H_{\theta_{0} 0}\right\}$. Then, by Lemma 1 ( $\S$ III), there exists a partial recursive function $b: \Theta \times N \rightarrow Q$ such that $|r(\theta)-b(\theta, n)|$ $<1 / n$ whenever $\phi\left\{H_{\theta}\right\} \neq 0$.

For $x \in H \cup \Theta$, let $s(x)=\sum_{i=1}^{|x|} x_{i} \cdot 2^{-i}$. The function $s: \Theta \rightarrow Q$ is recursive. 
We define sets $A(\theta)$ and $B(\theta)$ :

$$
\begin{aligned}
& A(\theta)=\{\sigma \in \Theta|s(\sigma)<b(\theta,|\sigma|)-2 /| \sigma \mid\}, \\
& B(\theta)=\{\sigma \in \Theta|s(\sigma)>b(\theta,|\sigma|)+2 /| \sigma \mid\},
\end{aligned}
$$

where $\sigma \in A(\theta)$ and $\sigma \in B(\theta)$ are partial recursive relations.

If $\phi\left\{H_{\theta}\right\} \neq 0$ and $x \in H$, we will show that

(a) $s(x)<r(\theta)$ iff $(\exists k)\left[x^{(k)} \in A(\theta)\right]$,

(b) $s(x)>r(\theta)$ iff $(\exists k)\left[x^{(k)} \in B(\theta)\right]$.

We prove (a). Suppose $(\exists k)\left[x^{(k)} \in A(\theta)\right]$. Since

$$
\sum_{i=1}^{k} x_{i} \cdot 2^{-i}<b(\theta, k)-\frac{2}{k}<r(\theta)-\frac{1}{k}
$$

we have

$$
\begin{aligned}
& \sum_{i=1}^{\infty} x_{i} \cdot 2^{-i}=\sum_{i=1}^{k} x_{i} \cdot 2^{-i}+\sum_{i=k+1}^{\infty} x_{i} \cdot 2^{-i} \\
& \leq \sum_{i=1}^{k} x_{i} \cdot 2^{-i}+\frac{1}{2^{k}}<r(\theta)-\frac{1}{k}+\frac{1}{2^{k}}<r(\theta) .
\end{aligned}
$$

Conversely, suppose $s(x)<r(\theta)$. Then there is a $k$ such that $s\left(x^{(k)}\right)+2 / k<$ $b(\theta, k)$. Then $x^{(k)} \in A(\theta)$. This proves (a); (b) is proved similarly.

Let $C(\theta)=A(\theta) \cup B(\theta) . \sigma \in C(\theta)$ is a partial recursive relation.

We will show that almost all $x \in H$, under the distribution $\psi_{1 / 2}$, have some initial segment in $C(\theta)$. This follows from

$$
\begin{gathered}
\psi_{1 / 2}\left\{x \in H \mid(\exists k)\left[x^{(k)} \in C(\theta)\right]\right\}=\psi_{1 / 2}\{x \in H \mid s(x)<r(\theta) \text { or } s(x)>r(\theta)\} \\
=1-\psi_{1 / 2}\{x \in H \mid s(x)=r(\theta)\}=1-\lim _{k \rightarrow \infty}\left(\frac{1}{2}\right)^{k}=1
\end{gathered}
$$

The motivation for what follows is that we choose successive bits according to $\psi_{1 / 2}$. (Th is corresponds to tossing a "fair" coin.) When, with $\sigma$ chosen, $s(\sigma)<r(\theta)$, we output 0 ; when $s(\sigma)>r(\theta)$, we output 1 . Then for the new $r(\theta)$, a new $\sigma$ is chosen.

Let $D(\theta)=\left\{\sigma \in C(\theta) \mid\right.$ if $\sigma^{\prime}<\sigma$, then $\left.\sigma^{\prime} \notin C(\theta)\right\}$, where $\sigma \in D(\theta)$ is a partial recursive relation.

For $\alpha \in \Theta, \alpha$ can be written uniquely as $\alpha=\gamma_{[1]} * \gamma_{[2]} * \ldots * \gamma_{[m]} * \delta$ where $\gamma_{[k]}$ is the sequence of bits that produces the $k$ th output bit, and $m$ is maximum with $m \geq 0$. More precisely, $\gamma_{[k]}=\sigma^{\left(k^{*}\right)}$ where

$$
k *\left[\theta^{(k-1)}\right]=(\mu k)\left[\sigma^{(k)} \in C\left(\theta^{(k-1)}\right)\right] .
$$

We must now define a recursive and monotone function $f: \Theta \rightarrow \Theta$ that, together with $\psi_{1 / 2}$, induces $\phi$. 
Let $f(\varnothing)=\varnothing$. For each successive input bit $i$, let $\delta^{\prime}=\delta * i$. If $\delta^{\prime} \notin$ $D\left[f\left(\gamma_{[1]} * \ldots * \gamma_{[m]}\right)\right]$, let $f(\alpha)=f\left(\gamma_{[1]} * \ldots * \gamma_{[m]}\right)$. If $\delta^{\prime} \in D\left[f\left(\gamma_{[1]} * \cdots * \gamma_{[m]}\right)\right]$, let $f(\alpha)=f\left(\gamma_{[1]} * \cdots * \gamma_{[m]}\right) * \epsilon$ where

$$
\boldsymbol{\epsilon}= \begin{cases}0 & \text { if } \delta^{\prime} \in A\left[f\left(\gamma_{[1]} * \ldots * \gamma_{[m}\right)\right], \\ 1 & \text { if } \delta^{\prime} \in B\left[f\left(\gamma_{[1]} * \ldots * \gamma_{[m}\right)\right] .\end{cases}
$$

The function $f$ is monotone. It is expressible by means of a multiple recursion and hence is recursive. We have that $\psi_{1 / 2}$ induces $\phi$. The theorem is proved.

In the following theorem, we note that $p$ need not be recursive.

Theorem 8. If $p$ is any real number with $0<p<1$, then $\psi_{p}$ induces $\psi_{1 / 2}$.

Proof. We mention by way of motivation that a " $p$ coin" is tossed in pairs until a nonhomogeneous pair is obtained. Then the output bit is, say, the last bit of the first pair for which that occurs. 0 and 1 will occur each with probability $1 / 2$.

We define subsets $A$ and $B$ of $\Theta$ :

$A=\{\sigma \in \Theta \mid(|\sigma|$ is even) and (the last two bits differ) and (no previous pair differ $)$ and $(\sigma|\sigma|=0)\}$,

$B=\{\sigma \in \Theta \mid(|\sigma|$ is even) and (the last two bits differ) and (no previous pair differ) and $(\sigma|\sigma|=1)\}$.

$A$ and $B$ are recursive sets. Let $D=A \cup B . D$ is recursive, as $A$ and $B$ are. Again, almost all $x \in H$ under $\psi_{p}$, have some initial segment in $D$.

For $\alpha \in \Theta, \alpha$ can be written uniquely with maximum $m$ in the form: $a=$ $\gamma_{[1]} * \ldots * \gamma_{[m]} * \delta$ where $m \geq 0$ and $\gamma_{[k]} \in D$ for $k \geq 1$.

We define a function $f: \Theta \rightarrow \Theta$ by $f(a)=\gamma_{[1]}^{\prime} * \ldots * \gamma_{[m]}^{\prime}$ where $\gamma_{[k]}^{\prime}$ is the last bit of $\gamma_{[k]}$. The function $f$ is recursive and monotone. Then $\psi_{p}$ induces $\psi_{1 / 2}$. This completes the proof of the theorem.

We summarize the previous two results.

Theorem 9. If $p$ is any real number sucb that $0<p<1$ and $\phi$ is a recursive distribution on $H, \cdot$ then $\psi_{p}$ induces $\phi$.

Proof. Theorems 7 and 8, followed by Theorem 2 .

Theorems 3 and 7 together show that the distributions induced by $\psi_{1 / 2}$ are those, and only those, that are recursive. We can ask: what distributions induce $\psi_{1 / 2}$ ? To that question we have the following partial answer.

Theorem 10. If $\psi$ is a recursive and atom-free distribution on $H$, then $\psi$ induces $\psi_{1 / 2}$.

Proof. We begin with a definition. 
Definition. For $x, y \in H, x$ is dominated by $y(x<y)$ if for some $i \geq 1$, $x^{(i-1)}=y^{(i-1)}$ and $x_{i}=0$ and $y_{i}=1$. $\prec$ is a total ordering on $H$. Recall that $s$ is the natural map of $H$ to the closed interval $[0,1]$. We have that $x \leq y$ iff $s(x) \leq s(y)$.

Let $\Lambda_{y}=\{x \in H \mid x<y\}$. For $A \subseteq \Theta$ and $X \subseteq H$, let $A * X=\{a * x \mid a \epsilon$ $A$ and $x \in X\}$.

By Lemma 1 ( $(\mathrm{III})$, we have, as $\theta * H_{a}=H_{\theta * a}$, that there exists a partial recursive function $w: \Theta \times \Theta \times N \rightarrow Q$ such that

$$
\left|\psi\left\{\theta * H_{\alpha}\right\} / \psi\left\{H_{\theta}\right\}-w(\theta, \alpha, n)\right|<1 / n
$$

whenever $\psi\left\{H_{\theta}\right\} \neq 0$.

We will obtain partial recursive approximations to $\psi\left\{\theta * \Lambda_{x}\right\} / \psi\left\{H_{\theta}\right\}$, which represents, whenever $\psi\left\{H_{\theta}\right\} \neq 0$, the relative measure of those sequences that follow $\theta$ and are dominated by $x$.

Define $u, v: \Theta \times \Theta \rightarrow \underline{Q}$ by

$$
\begin{aligned}
& u(\theta, \alpha)=\sum_{i=1}^{|\alpha|} \alpha_{i}\left[w\left(\theta, \alpha^{(i-1)} * 0,|\alpha| \cdot 2^{i}\right)-\frac{1}{|\alpha| \cdot 2^{i}}\right], \\
& v(\theta, \alpha)=1-\sum_{i=1}^{|\alpha|}\left(1-\alpha_{i}\right)\left[w\left(\theta, \alpha^{(i-1)} * 0,|\alpha| \cdot 2^{i}\right)-\frac{1}{|\alpha| \cdot 2^{i}}\right] .
\end{aligned}
$$

As $w$ is partial recursive, $u$ and $v$ are also.

We shall show that

(1) $u\left(\theta, x^{(n)}\right) \leq \psi\left\{\theta * \Lambda_{x}\right\} / \psi\left\{H_{\theta}\right\} \leq v\left(\theta, x^{(n)}\right)$,

(2) $\lim _{n \rightarrow \infty} u\left(\theta, x^{(n)}\right)=\psi\left\{\theta * \Lambda_{x}\right\} / \psi\left\{H_{\theta}\right\}=\lim _{n \rightarrow \infty} v\left(\theta, x^{(n)}\right)$.

First, for $x \in H$,

and

$$
\Lambda_{x}=\bigcup_{i=1 ; x_{i}=1}^{\infty} H_{x^{(i-1)} * 0}
$$

$$
H-\Lambda_{x}=\{x\} \cup \bigcup_{i=1 ; x_{i}=0}^{\infty} H_{x^{(i-1)} *_{1}} .
$$

From the first of these it follows that whenever $\psi\left\{H_{\theta}\right\} \neq 0$,

$$
\frac{\psi\left\{\theta * \Lambda_{x}\right\}}{\psi\left\{H_{\theta}\right\}}=\sum_{i=1}^{\infty} x_{i} \frac{\psi\left\{\theta * H_{x}^{(i-1)} * 0\right.}{\psi\left\{H_{\theta}\right\}}
$$

and from the second, as $\psi\{x\}=0$, that

$$
\frac{\psi\left\{\theta * \Lambda_{x}\right\}}{\psi\left\{H_{\theta}\right\}}=1-\sum_{i=1}^{\infty}\left(1-x_{i}\right) \frac{\psi\left\{\theta * H_{x^{(i-1)} * 1}\right\}}{\psi\left\{H_{\theta}\right\}}
$$


Then, for $n \geq 0$,

$$
\begin{aligned}
& \frac{\psi\left\{\theta * \Lambda_{x}\right\}}{\psi\left\{H_{\theta}\right\}}-u\left(\theta, x^{(n)}\right) \\
& =\sum_{i=1}^{\infty} x_{i} \frac{\psi\left\{\theta * H_{x^{(i-1)} * 0}\right\}}{\psi\left\{H_{\theta}\right\}}-\sum_{i=1}^{n} x_{i}\left[w\left(\theta, x^{(i-1)} * 0, n \cdot 2^{i}\right)-\frac{1}{n \cdot 2^{i}}\right] \\
& =\sum_{i=1}^{n} x_{i}\left(\delta_{n, i}+\frac{1}{n \cdot 2^{i}}\right)+\epsilon_{n}
\end{aligned}
$$

where

$$
\delta_{n, i}=\frac{\psi\left\{\theta * H_{x^{(i-1)} * 0}\right\}}{\psi\left\{H_{\theta}\right\}}-w\left(\theta, x^{(i-1)} * 0, n \cdot 2^{i}\right)
$$

and

$$
\epsilon_{n}=\sum_{i=n+1}^{\infty} \frac{\psi\left\{\theta * H_{x^{(i-1)} * 0}\right\}}{\psi\left\{H_{\theta}\right\}}
$$

From the definition of $w,\left|\delta_{n, i}\right|<1 /\left(n \cdot 2^{i}\right)$, and since

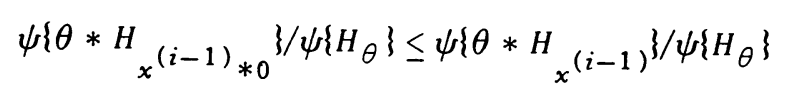

and $\psi$ is atom-free, $\lim _{n \rightarrow \infty} \epsilon_{n}=0$. Hence $0<\delta_{n, i}+1 /\left(n \cdot 2^{i}\right)<2 /\left(n \cdot 2^{i}\right)$. Then

$$
\begin{aligned}
0 & <\frac{\psi\left\{\theta * \Lambda_{x}\right\}}{\psi\left\{H_{\theta}\right\}}-u\left(\theta, x^{(n)}\right)=\sum_{i=1}^{n} x_{i}\left(\delta_{n, i}+\frac{1}{n \cdot 2^{i}}\right)+\epsilon_{n} \\
& <\sum_{i=1}^{n}\left(\delta_{n, i}+\frac{1}{n \cdot 2^{i}}\right)+\epsilon_{n}<\sum_{i=1}^{n} \frac{2}{n \cdot 2^{i}}+\epsilon_{n}<\frac{2}{n}+\epsilon_{n} .
\end{aligned}
$$

This proves that

$$
\lim _{n \rightarrow \infty} u\left(\theta, x^{(n)}\right)=\frac{\psi\left\{\theta * \Lambda_{x}\right\}}{\psi\left\{H_{\theta}\right\}} .
$$

The similar fact for $v$ instead of $u$ is proved similarly.

We next show that

$$
\psi\left\{x \in H \mid \psi\left\{\Lambda_{x}\right\}<1 / 2\right\}=1 / 2 .
$$

We have that $x<y$ iff $s(x) \leq s(y)$. Let $p(x)=\psi\left\{s^{-1}[0, x]\right\}$ for $0 \leq x \leq 1 . p(0)$ $=0$ because $\psi$ is atom-free, and $p(1)=1$ because $\psi\{H\}=1 . p$ is monotone nondecreasing because $\psi$ is nonnegative, and $p$ is continuous because $\psi$ is atomfree. Let 


$$
\begin{aligned}
& K^{-}=\{x \in[0,1] \mid p(x)<1 / 2\}, \\
& K^{0}=\{x \in[0,1] \mid p(x)=1 / 2\}, \\
& K^{+}=\{x \in[0,1] \mid p(x)>1 / 2\},
\end{aligned}
$$

$0 \in K^{-}$and $1 \in K^{+}$. Because $p$ is continuous, $p$ must take on every value between 0 and 1 . Then $K^{0}$ is not empty, and it is closed. Let $u=g l b K^{0}$ and $v=\operatorname{lub} K^{0}$. Then

$$
K^{-}=[0, u), \quad K^{0}=[u, v], \quad K^{+}=(v, 1] .
$$

Now, because $\psi$ is atom-free,

$$
\begin{gathered}
\psi\left\{x \in H \mid \psi\left\{\Lambda_{x}\right\}<1 / 2\right\}=\psi\left\{x \in H \mid \psi\left\{s^{-1}[0, s(x)]\right\}<1 / 2\right\} \\
=\psi\left\{x \in H \mid s(x) \in K^{-}\right\}=\psi\{x \in H \mid s(x) \in[0, u)\} \\
\left.=\psi\left\{s^{-1}[0, u)\right\}=\psi\left\{s^{-1}[0, u]\right\}=p^{\prime} u\right)=1 / 2
\end{gathered}
$$

because $u \in K^{0}$.

This proves that

$$
\psi\left\{x \in H \mid \psi\left\{\Lambda_{x}\right\}<1 / 2\right\}=1 / 2 .
$$

A similar proof shows that

$$
\psi\left\{x \in H \mid \psi\left\{\Lambda_{x}\right\}>1 / 2\right\}=1 / 2 .
$$

It follows that

$$
\psi\left\{x \in H \mid \psi\left\{\Lambda_{x}\right\}=1 / 2\right\}=0 \text {. }
$$

Finally, define a function $g: \Theta \times \Theta \rightarrow \Theta$ by:

$$
g(\theta, \alpha)= \begin{cases}\varnothing \quad \text { if } u(\theta, \beta) \leq 1 / 2 \leq v(\theta, \beta) \text { for all } \beta \leq \alpha, \\ \{0\} \quad \text { if } \min _{\beta \leq \alpha}|v(\theta, \beta)|<1 / 2, \\ \{1\} \quad \text { if } \max _{\beta \leq \alpha}|u(\theta, \beta)|>1 / 2 .\end{cases}
$$

We have that $g$ is partial recursive; it is defined for all $\alpha \in \Theta$ whenever $\psi\left\{H_{\theta}\right\}$ $\neq 0$; and it is monotonic in $\alpha$. For $x \in H$ such that $\psi\left\{\theta * \Lambda_{x}\right\} / \psi\left\{H_{\theta}\right\}<1 / 2$, there is an $n$ such that $g\left(\theta, x^{(n)}\right)=\{0\}$; and for $x \in H$ such that $\psi\left\{\theta * \Lambda_{x}\right\} / \psi\left\{H_{\theta}\right\}$ $>1 / 2$, there is an $n$ such that $g\left(\theta, x^{(n)}\right)=\{1\}$.

Now, $g$ can be used to define a monotone partial recursive function $b: \Theta \rightarrow$ $\Theta$ such that for almost all inputs $x \in H$, the re is a sequence $x_{[1]}, x_{[2]}, \cdots$, such that $x=x_{[1]} * x_{[2]} * \cdots$ and, letting $x^{[n]}=x_{[1]} * \cdots * x_{[n]}$, we have $b\left(x^{[n]} * \tau\right)=b\left(x^{[n]}\right) * g\left(x^{[n]}, \tau\right)$ tor all $n \geq 0$ and $\tau \leq x_{[n+1]}$.

Since $b$ is partial recursive, there exists a recursive relation $R$ on $\Theta \times N$ and a recursive function $U: N \rightarrow \Theta$ such that $b(\theta)=U[(\mu n) R(\theta, n)]$.

Let the function $j: \Theta \times N \rightarrow \Theta$ be defined by 


$$
j(\theta, n)= \begin{cases}h(\theta) & \text { if }(\exists m)[m \leq n \text { and } R(\theta, m)] \\ \varnothing & \text { otherwise. }\end{cases}
$$

$j$ is a recursive function. Let the function $f: \Theta \rightarrow \Theta$ be defined by

$$
f(\theta)=\max _{0 \leq i \leq|\theta|} j\left(\theta^{(i)},|\theta|\right) .
$$

Then $f$ is recursive and it can be seen that it is monotone. $(\psi, f)$ induces $\psi_{1 / 2}$. This proves the theorem.

We have immediately the following generalization of Theorem 8 .

Corollary. Any recursive atom-free distribution induces any recursive distribution.

Proof. Theorems 7 and 10, followed by Theorem 2 .

We define a partial order on distributions. We will say for $\psi$ and $\phi$ distributions on $H$, that $\phi \precsim \psi$ if $\psi$ induces $\phi$. We say that $\phi \sim \psi$ if $\phi \preccurlyeq \psi$ and $\psi \lesssim \phi$. And $\phi \prec \psi$ if $\phi \lesssim \psi$ and not $\psi \sim \phi$.

The partial order $\lesssim$ is reflexive and transitive. Under the equivalence relation $\sim$, we can refer to the equivalence class of $\psi$ as $[\psi]$. The partial order extends to the equivalence classes.

$\left[\psi_{1 / 2}\right]$ consists of the recursive atom-free distributions on $H$. It is true that $\left[\psi_{1 / 2}\right]$ is not lowest in the partial order, as $\psi_{1 / 2}$ induces (Theorem 7), and is not induced by (Theorem 6), recursive, non-atom-free distributions. And from Theorems 1,3 , and 8 , it follows that if $p, 0<p<1$, is a nonrecursive real number, then $\left[\psi_{1 / 2}\right] \prec\left[\psi_{p}\right]$.

There remain open questions. We do not know, for instance, whether $\psi_{p}$ induces $\psi_{q}$ when $q$ is recursive in $p$, though the converse is strongly indicated by ideas similar to those used in the proof of Theorem 3.

In the more general framework, we can ask whether every atom-free distribution is an element of some $\left[\psi_{p}\right]$, that is, can such a distribution be characterized for the partial order by a single real number? Alternatively, this would be precluded by a result that is entirely possible: there exist atom-free distributions that induce no recursive atom-free distribution. As distributions may have much more structure than number-theoretic functions, we must count the last of these as more likely. On these questions hinge whether the partial order on distributions is equally rich, or richer, than that of degrees of unsolvability.

\section{BIBLIOGRAPHY}

1. Alonzo Church, An unsolvable problem of elementary number theory, Amer. J. Math. 58 (1936), 345-363. 
2. Martin Davis, Computability and unsolvability, McGraw-Hill, New York, 1958. MR 23 \#A 1525.

3. K. de Leeuw, E. F. Moore, C. E. Shannon and N. Shapiro, Computability by probabilistic machines, Automata Studies, Ann. of Math. Studies, no. 34, Princeton Univ. Press, Princeton, N. J., 1956, pp. 183-212. MR 18, 104.

4. P. R.Halmos, Measure theory, Van Nostrand, Princeton, N. J., 1950. MR 11, 504.

5. S. C. Kleene, Introduction to metamathematics, Van Nostrand, Princeton, N. J., 1952. MR 14, 525.

6. J. R. Myhill, Criteria of constructabllity for real numbers, J. Symbolic Logic 18 (1953), 7-10. MR 14, 938.

7. H. G. Rice, Recursive real numbers, Proc. Amer. Math. Soc. 5 (1954), 784-791. MR 16, 104.

8. Raphael Robinson, (review) Rósza Peter, Rekursive Functionen, J. Symbolic Logic 16 (1951), 182.

9. Emst Specker, Nicht Konstructive Beweisbare Sätze Der Analysis, J. Symbolic Logic 14 (1949), 145-158. MR 11, 151.

26 WEST 10TH STREET, NEW YORK, NEW YORK 10011 